\title{
Degradation of Cellulose Acetate-Based Materials: A Review
}

\author{
Juergen Puls · Steven A. Wilson • Dirk Hölter
}

Published online: 20 November 2010

(C) The Author(s) 2010. This article is published with open access at Springerlink.com

\begin{abstract}
Cellulose acetate polymer is used to make a variety of consumer products including textiles, plastic films, and cigarette filters. A review of degradation mechanisms, and the possible approaches to diminish the environmental persistence of these materials, will clarify the current and potential degradation rates of these products after disposal. Various studies have been conducted on the biodegradability of cellulose acetate, but no review has been compiled which includes biological, chemical, and photo chemical degradation mechanisms. Cellulose acetate is prepared by acetylating cellulose, the most abundant natural polymer. Cellulose is readily biodegraded by organisms that utilize cellulase enzymes, but due to the additional acetyl groups cellulose acetate requires the presence of esterases for the first step in biodegradation. Once partial deacetylation has been accomplished either by enzymes, or by partial chemical hydrolysis, the polymer's cellulose backbone is readily biodegraded. Cellulose acetate is photo chemically degraded by UV wavelengths shorter than $280 \mathrm{~nm}$, but has limited photo degradability in sunlight due to the lack of chromophores for absorbing ultraviolet light. Photo degradability can be significantly
\end{abstract}

J. Puls

vTI Institute of Wood Technology and Wood Biology,

Hamburg, Germany

e-mail: juergen.puls@vti.bund.de

S. A. Wilson $(\square)$

Eastman Chemical Company, Kingsport, TN, USA

e-mail: sawilson@eastman.com

D. Hölter

Rhodia Acetow GmbH, Freiburg, Germany

e-mail: dirk.hoelter@eu.rhodia.com enhanced by the addition of titanium dioxide, which is used as a whitening agent in many consumer products. Photo degradation with $\mathrm{TiO}_{2}$ causes surface pitting, thus increasing a material's surface area which enhances biodegradation. The combination of both photo and biodegradation allows a synergy that enhances the overall degradation rate. The physical design of a consumer product can also facilitate enhanced degradation rate, since rates are highly influenced by the exposure to environmental conditions. The patent literature contains an abundance of ideas for designing consumer products that are less persistent in the outdoors environment, and this review will include insights into enhanced degradability designs.

Keywords Biodegradation - Photo degradation . Disintegration

\section{Introduction}

Cellulose acetate (CA) is a modified natural polymer with a wide range of properties [1,2]. Although CA can be produced with a range of degrees of substitution (DS), the most common level is a DS of 2.5 due to good solubility in common solvents, molecular weights, and melt properties. These properties facilitate CA's use in a variety of consumer products including textiles, plastics, films, and cigarette filters that can be discarded and become litter. Considering that the global production for CA materials was over 800,000 metric tons per year in 2008 (Table 1), and many items are disposed of as surface litter or into composting facilities, it is important to understand the fate of discarded CA-based products. An increased awareness of routes of degradation could prove useful to understand the ultimate environmental impact. 
Table 1 Global production of cellulose acetate-based products

\begin{tabular}{llc}
\hline $\begin{array}{l}\text { Cellulose diacetate } \\
\text { materials }\end{array}$ & $\begin{array}{l}\text { Degree of } \\
\text { substitution } \\
\text { (DS) }\end{array}$ & $\begin{array}{l}\text { 2008 Global } \\
\text { production } \\
\text { (metric tons) }\end{array}$ \\
\hline Coatings, plastics and films & 2.5 & 41,000 \\
Textile fiber & 2.5 & 49,000 \\
Filter tow & 2.5 & 690,000 \\
LCDs, photo films, and textiles & 3.0 & 41,000 \\
\hline
\end{tabular}

Source Chemical Economics Handbook (CEH) Marketing Report

Initially the assessments of the biodegradability of CA reached the incorrect conclusion that the polymer is not biodegradable, due to evaluations being performed only with cellulose degrading organisms like fungi [3]. Later the importance of the deacetylation step was shown, when it was learned that acetyl esterase enzymes are common in microorganisms. Currently CA is generally recognized as a biodegradable polymer within the scientific community [48]. Recent work has allowed a considerable increase in the knowledge of the enzymology of non-substituted and acetylated polysaccharides, thus illuminating the biodegradation mechanism.

Photo degradation is another common mechanism for many polymers. Although CA polymer alone has limited photo degradation in sunlight, many consumer products have additives that allow enhanced photo degradation. Titanium dioxide is commonly added to enhance the whiteness of CA materials, and is a photo oxidation catalyst that causes degradation in sunlight.

The final section of this review will cover design approaches that propose to further enhance the degradation mechanisms. Research has shown that the combination of both bio and photo degradation allows a synergism that improves the overall degradation rate. This synergy is a result of photo degradation causing pitting and thereby increasing the material's surface area, and enhancing biodegradation. Research has shown that the environmental conditions have a strong impact on a material's degradation rate. The patent literature contains many ideas disclosed for making consumer products which can be designed to optimize the degradation environment. A review of key patent disclosures will illustrate highlights of this diversity of ideas for enhancing degradation rates.

This paper will review each topic and bring together the subject, allowing a more complete understanding of the ultimate environmental impact of CA based products.

\section{Biological Degradation}

The early research into the biodegradation of CA polymer produced some conflicting conclusions. Some researchers reported that natural organisms could not degrade CA with a DS of greater than $1.5[3,9]$, while other researchers determined that CA with DS of 2.5 had limited utility due to its degradation $[10,11]$. Later experiments identified that the key mechanism for degradation is an initial deacetylation step by chemical hydrolysis and acetylesterases, thereby allowing the degradation of the cellulose backbone with cellulase [12]. In hindsight, perhaps it is self-evident that the mechanisms for biodegradation should be different for cellulose versus CA polymers.

In 1957 Reese published the first study on the biological degradation of various cellulose derivatives and concluded that just one acetyl per glucose was sufficient to ensure resistance to degradation by cellulase enzymes [9]. There was no deacetylation step in this study. In 1972 EPA Report No EPA-R2-72-046 was published investigating the biodegradability of packaging plastics. The study consisted of exposing the polymers to fungi using ASTM Method D1924-63 and judged the growth rates after exposure times of up to 3 weeks [3]. Under these limited test conditions, the authors reached the conclusion that $\mathrm{CA}$ is not biodegradable.

Biodegradation was shown in early investigations, where CA-based reverse osmosis membranes of DS 2.5 were incubated with a variety of microorganisms [11]. Some sources of microorganisms were more successful in attacking the CA membranes than others. After 2 months of incubation some CA samples lost their semi-permeability with up to $50 \%$ loss of acetyl groups.

It is noteworthy that there has been some debate about the definition of biodegradation. One view is that biodegradation is defined as microbial initiated conversion of a substrate in a biologically active environment into carbon dioxide (aerobically), methane (anaerobically), cell wall material, and other biological products. Another view is a requirement of a certain rate of degradation, such as weight loss versus time.

One novel study was the findings by forensic science researchers Northrop and Rowe in 1987, who studied the effect of the soil environment on the biodeterioration of man-made textiles [13]. They found that cellulose acetate fibers were significantly deteriorated after 2 months in moist soil and were completely destroyed after 4-9 months. In this study, the other synthetic textile fibers (nylon, polyester and acrylics) showed no significant changes at the end of the 12 month study.

Since these early mixed results, several studies have confirmed that CA is indeed biodegraded in a natural environment as measured by a variety of methods. One of the more convincing degradation studies was the aerobic biodegradation of radiolabelled CA by Komarek, Gardner, and Buchanan where they monitored the evolution of $\mathrm{CO}_{2}$ from in vitro samples with the acetyl carbons labeled with 
${ }^{14} \mathrm{C}$ [5]. The study compared CA with degrees of substitutions of 1.85, 2.07 and 2.57, and found the biodegradation rates were reduced, but not inhibited, by the higher levels of acetyl (Fig. 1).

Buchanan, Gardner, and Komarek in 1993 [4] studied two aerobic assay systems for degrading CA films: an in vitro enrichment cultivation technique and an activated sludge wastewater treatment system. The enrichment culture was able to degrade CA films within 2-3 weeks, as indicated by $67 \%$ weight loss. The industrial wastewater treatment system provided the same degradation, however, it occurred at slower rates with 10 weeks required for significant changes in the films. The authors stressed the point that the accelerated degradation systems only included an increase in concentration and not a change in the abilities of the naturally occurring microorganisms; thus when CA materials are placed in a natural environment the potential for biodegradation is maintained and only the speed of degradation is changed.

CA has also been shown to be degraded under anaerobic conditions. Rivard et al. in 1992 [7] studied CA of increasing DS (0.82-2.4) by incubating them with a special culture for a period of 98 days, and found that CA of DS $<1.25$ were significantly degraded. The samples with DS $>1.7$ were also degraded, but to a lesser extent.

In 1993, Gu, Eberiel, McCarthy and Gross studied the biodegradability of CA films with DS between 1.7 and 2.5 under controlled composting conditions. The materials were exposed to biologically active laboratory aerobic test vessels at $53{ }^{\circ} \mathrm{C}$ [8]. They found that the films completely disappeared after incubation for 7 and 18 days, respectively. In a related paper, the researchers evaluated the above degraded films by gel permeation chromatography

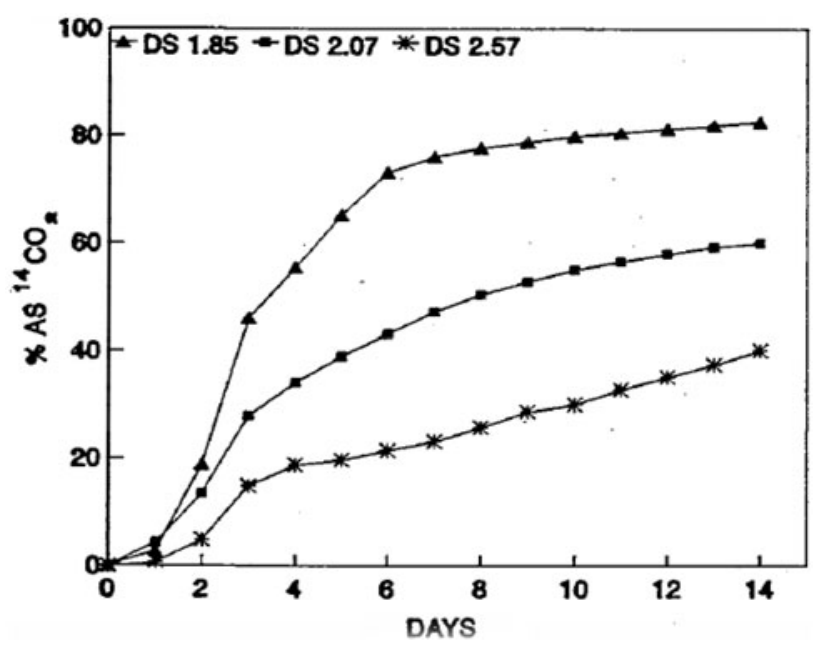

Fig. 1 Biodegradation of cellulose $\left[1-{ }^{14} \mathrm{C}\right]$-acetates (DS 1.85, 2.07, and 2.57) as indicated by the percent of labeled carbon metabolized to ${ }^{14} \mathrm{CO}_{2}$
(GPC), proton nuclear magnetic resonance $\left({ }^{1} \mathrm{H}\right.$ NMR), and scanning electron microscopy (SEM) to understand the degradation mechanisms [14]. Noteworthy observations were the pitting of the films by microbes and the limited changes in DS and molecular weights as the polymer degraded. It was proposed that the random cleavage of the polymer caused low molecular weight degradation products that would diffuse away from the bulk materials and be rapidly assimilated by the microorganisms. In the same study $\mathrm{Gu}$ et al. disclosed the results of placing cellophane and a CA film with $\mathrm{DS}=1.7$ in a bioreactor under anaerobic conditions. After 30 days, the CA film had been degraded completely [8]. The authors of this study stressed that both aerobic and anaerobic microorganisms are known to produce the complete set of hydrolases, including esterases, which are necessary to degrade naturally occurring acetylated polysaccharides. This includes acetyl-4$O$-methylglucuronoxylan, acetylgalactoglucomannan, and chitin. In the same group, Nelson et al. [15] isolated a series of CA degrading Pseudomonas strains as a part of the degradation study. In 1994, Gu et al. [16] demonstrated that the time required for CA degradation under laboratory composting conditions was dependent on the variation in the mixture composition of the compost especially the water content. These researchers also determined the DS of the CA material after 35-50\% weight loss and found that there was no significant change in DS of the residual material. From this result they concluded that the degradation was completely biological and not induced by a chemical deacetylation.

Sakai et al. [17] found two strains of the bacterium Neisseria sicca that degrade cellulose acetate with DS 1.8 and DS 2.3. The highest degradation rates reported were 60 and $45 \%$, both within 20 days. Alcaligenes xylosoxidans was identified as another CA degrading microorganism.

CA fibers with DS 2.45 also proved to be anaerobically degradable measured according to ASTM D 5210-91 and ISO 11734 [18]. The determination of anaerobic degradability is based on the liberation of biogas using diluted digested sludge as the inoculums. Note the principal difference between the two test methods is the buffer system with the ASTM method using a bicarbonate buffer versus the ISO method using a hydrogen phosphate buffer. The study demonstrated that after 3 weeks in the ASTM medium $60-70 \%$ of the initial CA as well as of the reference material cotton was degraded. In contrast to cotton where degradation had already begun after 4 days, the lag time for CA was 11 days, followed by similar degradation kinetics. The anaerobic degradation of cigarette filter tips made with CA tow with DS 2.45 was verified in the paper of Zenjian et al. [19].

Within their review on cellulose ester performance and application, Edgar and coworkers identified commercially 
used cellulose acetates to be biodegradable, especially under composting conditions [20]. It is worth noting that composting is a sequence of appearance of microorganisms with a constant shift from the acid to the neutral and alkaline $\mathrm{pH}$ region, in which the $\mathrm{pH}$ may reach values as high as $\mathrm{pH}$ 9. These are also the conditions, under which at least partly, acetyl substituents start to be cleaved off. The temperature in naturally progressing composting may rise up to $70{ }^{\circ} \mathrm{C}$ and higher by self-heating, which are intended composting conditions for the sterilization of the soil [21], (see Fig. 2).

In an attempt to degrade filter tow with a DS of 2.5 under natural composting conditions at the laboratory scale, a temperature of $75{ }^{\circ} \mathrm{C}$ was reached. In this experiment the thermophilic phase $\left(>50^{\circ} \mathrm{C}\right)$ lasted for $6-12$ days [6]. After one cycle, the samples showed a weight loss of $8 \%$. But after a second run-through, the filaments of the samples had disappeared without any detectable trace. Gardner et al. examined CA films in another laboratory scale test with a thermophilic phase of approximately 5 days. They found, that the weight loss is highly dependent upon DS with a significantly retarded degradation at DS greater than 2.1 [6]. It should be noted that, in contrast to the laboratory tests, in commercial composting the thermophilic phase normally lasts longer and the compost is mechanically treated.

A DS 1.7 CA film was completely disintegrated after 7 days composting, whereas a DS $2.5 \mathrm{CA}$ film required 18 days biological degradation of cellulose acetate. The biodegradability of CA with DS 2.5 was also assayed applying the modified Sturm method for aerobic degradability. Within 9 weeks the degradation rate varied between 51 and $87 \%$, based on $\mathrm{CO}_{2}$-liberation [22].

Van der Zee and coworkers compared CA biodegradability under laboratory controlled composting conditions

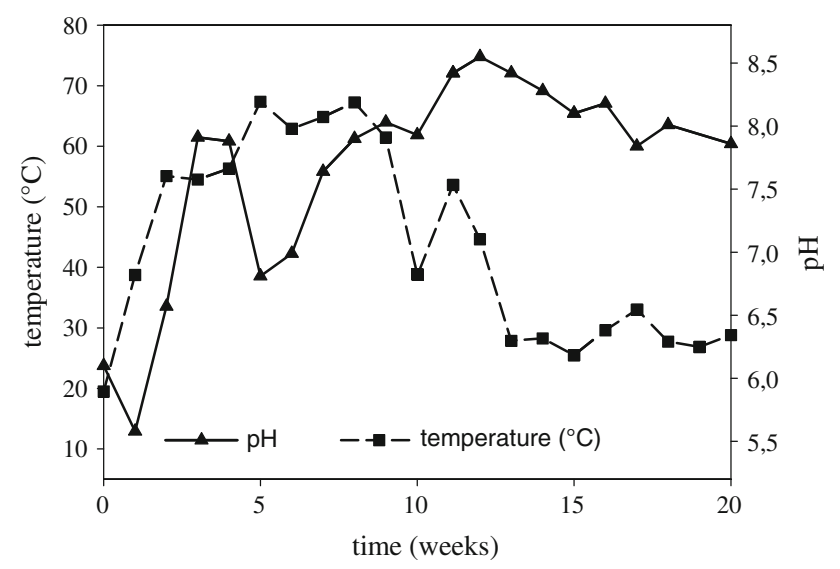

Fig. 2 Changes of temperature and $\mathrm{pH}$ value during composting with difference air streams (from [21] modified) with an aquatic biodegradation test [23]. It was found that CA was indeed biodegraded under composting conditions; however surprisingly in their work CA biodegradation was not observed in the aquatic test.

One of the earliest approaches to making CA-based plastics with enhanced biodegradability was published by Ach in 1993 [24]. Biodegradable plastics were prepared and evaluated, using low-molecular weight liquid additives that are biodegradable and blended with $\mathrm{CA}$ at a 2:1 ratio.

In 2000, Yoshioka and Shiraishi [25] described their development of biodegradable plastics based upon the reaction of cellulose acetate with dibasic acid anhydrides and monoepoxides. In addition to good thermoplastic properties, they found better biodegradability compared to unmodified cellulose diacetate.

The patent literature is a source of information on how materials or products might be changed to enhance biodegradation. For example, commercial fibers are made with CA with a DS 2.5, but research has shown that CA polymer with lower DS biodegrades faster. In US Patent 5,427,852 Teufel [26] discloses hydrolyzing the surface of CA fibers to enhance the biodegradation. In US Patent 5,462,801 Willmund [27] discloses an improved rate of degradation by adding cellulose chain-splitting enzymes to the fiber's surface. In US Patent 5,495,860 Teufel discloses CA fibers containing nitrogenous organic compounds which enhance degradation after microorganisms form basic decomposition products [28]. These basic compounds, e.g., ammonia, are said to enhance the hydrolysis of acetyl groups and accelerate degradation.

In US Patent 5,512,230 Cannon discloses that low DS CA fibers can be spun on commercial acetone-solvent spinning equipment by using more water in the acetone solvent and thus shift the solvent's polarity [29]. This invention allows the spinning of CA polymer with DS 1.9 to 2.2 which are not readily soluble in acetone. Using this process one could make cigarette filters with enhanced biodegradation without using polymer additives. In related research, Buchanan et al. [30] explored the miscibility and biodegradation of CA blends with DS of 2.0 and 2.5 for plastics. They found that the optically clear blends with useful physical properties would also have good biodegradability. The addition of a polyethylene glycol (MW 400) plasticizer also disclosed to enhance the degradation rate.

Itoh et al. [31], disclose in US Patent 5,478,386 that a cellulose ester blend with at least $10 \%$ low DS CA (DS $<2.15$ ) with the CA polymer having a ratio of sulfate to residual alkali metals or alkali earth metals of 0.1 to 1.1 would provide enhanced biodegradation. The residual sulfate groups are the result of using sulfuric acid using the esterification process. 


\section{Enzymology of Cellulose Acetate Biodegradation}

In recent years there has been significant research into the enzymes that degrade CA. Non-derivatized cellulose can be hydrolyzed very efficiently by the cellulolytic enzyme system consisting of several cellobiohydrolases $(\mathrm{CBH})$, acting from the reducing and non-reducing end of the cellulose chain, and endoglucanases (EG) which acts randomly within the chain. This enzyme system is being used commercially for the production of 2 nd generation ethanol from lignocellulosic material.

Cellobiohydrolases are able to attack amorphous and crystalline cellulose regions, but are rather sensitive to all types of cellulose substituents, including acetyl groups, due to the fact that the active site of this enzyme class is in a tunnel formed by stable surface loops [32]. The tunnelshaped active site restricts the hydrolysis to occurring at the ends of the cellulose chain. Because hydrolysis continues progressively, it may be stopped at the first substitution encountered. Indeed cellobiohydrolases are believed to be unable to attack cellulose derivatives, and the existence of the active site inside a tunnel could explain that cellulose derivatives would get stuck, depending on their size and their charge (Fig. 3).

Cellobiohydrolase I (CBHI) cleaves B-1,4-glycosidic bonds with retention of configuration, yielding the $B$-anomer as reaction product [33]. It starts its attack from the reducing end, whereas cellobiohydrolase II (CBHII),
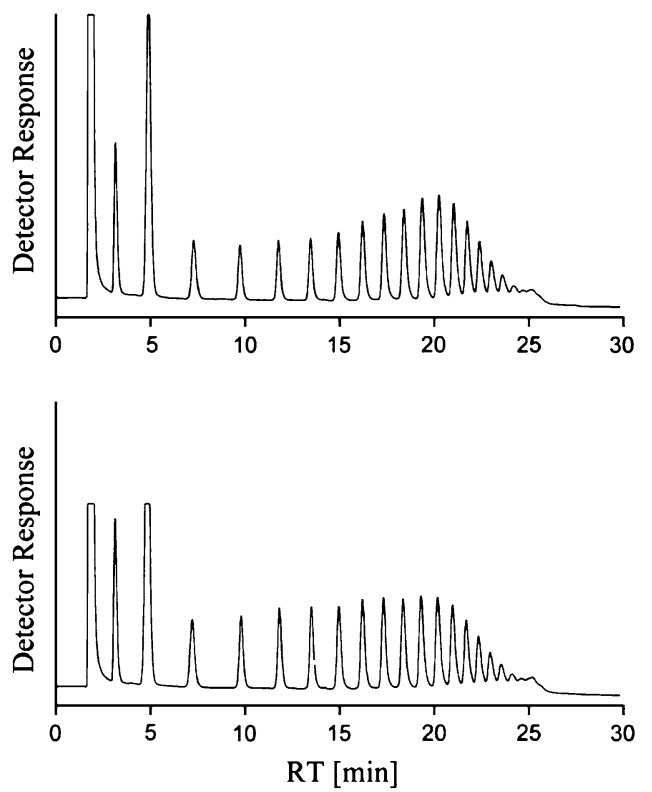

Fig. 3 Water-soluble CA (DS 0.7) oligosaccharides ( DP12) after incubation with cellobiohydrolases $(\mathrm{CBH})$ of Trichoderma reesei, as analyzed by anion exchange chromatography in $\mathrm{NaOH}$ medium combined with pulsed amperometric detection. Upper left $\mathrm{CBH}$ I alone. Upper right $\mathrm{CBH}$ I and $\mathrm{CBH}$ II acting together from reducing and non-reducing end. Bottom left $\mathrm{CBH}$ II alone. Bottom right attacks the cellulose chain from the non-reducing end. CBHII inverts the configuration at the glycosidic bond, so that the first-formed cellobiose is the $\alpha$-anomer [34]. Taking the structure of cellobiohydrolases into account it was no wonder that a mixture of pure CBHI:CBHII:EGI:E$\mathrm{GII}=6: 2: 1: 1$ was unable to degrade CA DS 2.5, since acetyl cleaving enzymes were not present in this artificial mixture [35]. However, $78 \%$ of cellophane and $43 \%$ of unbleached kraft pulp were hydrolyzed within $6 \mathrm{~h}$.

In contrast to exo-acting cellobiohydrolases, endoglucanases are only able to degrade disordered, amorphous regions of cellulose, cutting at internal glycosidic bonds [36]. However, advantageously their active site is more open arranged and placed in a cleft, allowing a random hydrolysis of the cellulose chain [37] (Fig. 4). Correspondingly endoglucanase-initiated hydrolysis of cellulose derivatives is possible, the extent not only being dependent on the degree of substitution, but also on the substituent distribution along the cellulose chain [38] and the architecture of the enzyme protein [39]. For an effective hydrolysis of CA, endoglucanase would be the most favored especially if non-substituted or partly substituted anhydroglucose units are present (Table 2).

In a careful study, the ability of TR Cel7B (endoglucanase I from Trichoderma reesei) to fragment CA of increasing DS was described by Saake et al. [40]. The degree of CA substitution ranged from 0.9 to 2.9, and the degree of polymerization varied from 16,000 to 201,000 .
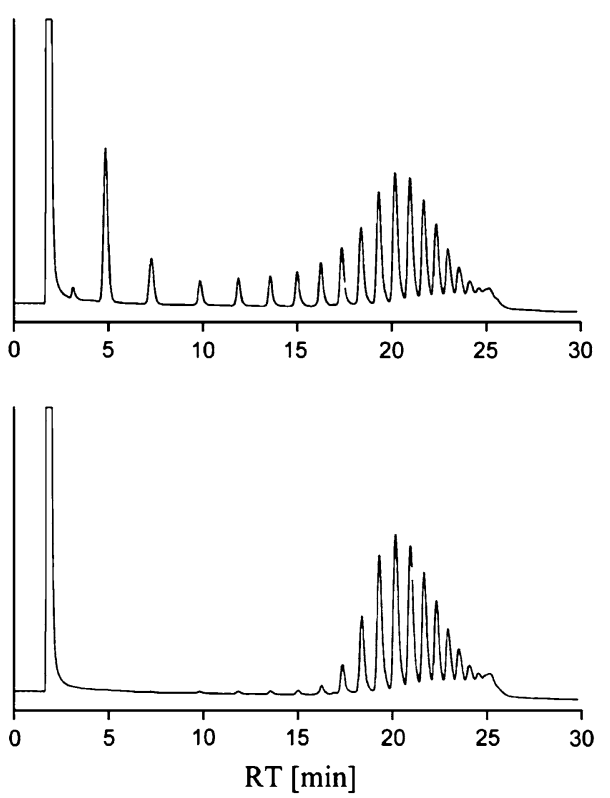

Reference substrate. Retention time (RT) of substrates and products: Glucose $=3 \mathrm{~min}$; cellobiose $=5 \mathrm{~min}$; cellotriose $=7 \mathrm{~min}$; cellotetraose $=10 \mathrm{~min}$; cellopentaose $=12 \mathrm{~min} ;$ cellohexaose $=14 \mathrm{~min}$; celloheptaose $=15 \mathrm{~min} ; \quad$ cellooctaose $=16 \mathrm{~min} ; \quad$ cellononaose $=$ $18 \mathrm{~min}$; cellodecaose $=19 \mathrm{~min}$; cello-undecaose $=20 \mathrm{~min}$; cellododecaose $(\mathrm{DP} 12)=21 \mathrm{~min}$ 


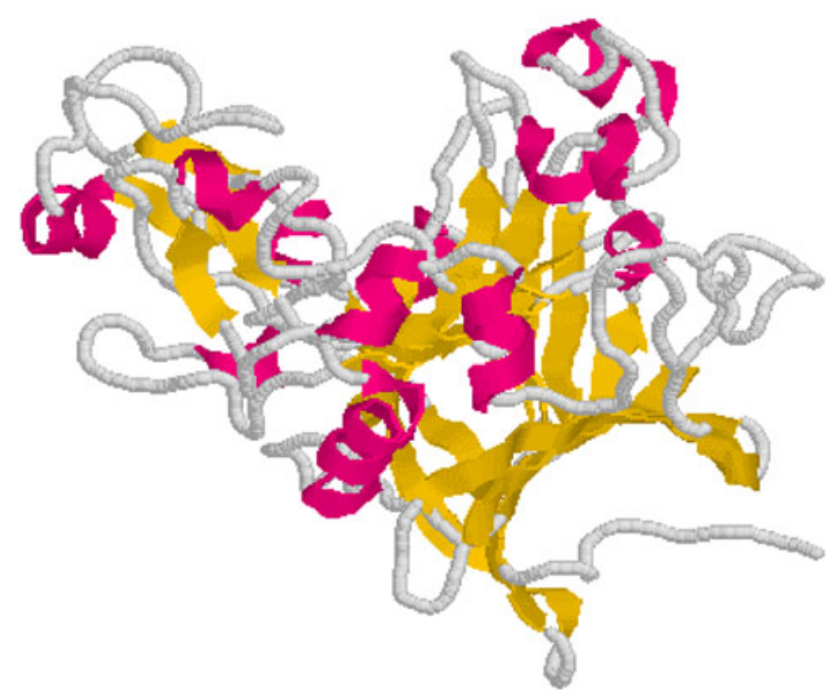

Fig. 4 Active site topology of endoglucanase Cel7B from Humicola insolens [89]

Table 2 Potential of endoglucanase Cel7B to degrade cellulose acetate dependent on the degree of substitution (DS)

\begin{tabular}{llc}
\hline DS & DPw & \\
\cline { 2 - 3 } & Starting material & Cel7B-fragmented \\
\hline 0.9 & 31 & 4 \\
1.2 & 85 & 5 \\
1.6 & 138 & 27 \\
1.9 & 189 & 100 \\
2.5 & 316 & 306 \\
2.9 & 387 & 394 \\
\hline
\end{tabular}

All samples were intensively incubated with an endoglucanase free of acetylesterase activity, and the degradation was monitored using size exclusion chromatography (SEC). The DS 0.9 sample was readily degraded from DP 31 to DP 4 . With up to DS 1.7 a $50 \%$ reduction in DP could be observed under the experimental conditions. For the DS 1.9 sample the DP reduction was considerably retarded, namely from DP $=189$ to 100 , while CA of DS 2.5 was more or less resistant to $\mathrm{Cel} 7 \mathrm{~B}$ action (DP reduction from 316 to 306). The accessibility for the endoglucanase was a clear function of the DS distribution pattern. The decrease in chain reduction was certainly caused by two factors: the water-insolubility of the material, and the shielding by increased amounts of acetyl substituents. In a separate study, an endoglucanase from $N$. sicca SB did not hydrolyze the CA main chain with DS of 1.77 , but when the degree of substitution was low $(<1.0)$, the endoglucanase hydrolyzed the CA main chain [40].

It has been known for a long time that in addition to cellulose main chain degrading enzymes, acetyl esterases (a)

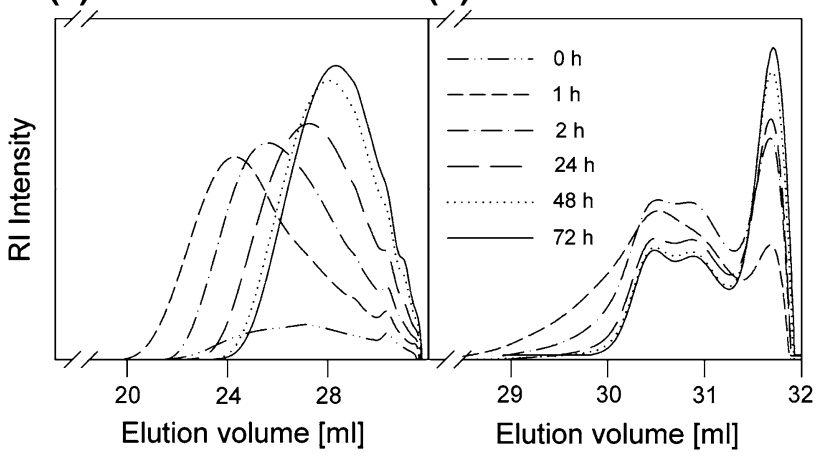

Fig. 5 Size exclusion chromatography (SEC) profiles of watersoluble cellulose acetate (DS 0.7) fragmented by endoglucanase Cel7B alone (a) and an Aspergillus enzyme mix including acetylesterase activity (b). Note the different scale in RT of Fig. 5a, b due to the extended degradation by the endoglucanase and acetylesterase mix (Fig. 5b) in contrast to fragmentation by endoglucanase alone (Fig. 5a)

play a key role in the biological degradation of CA. Reese in 1957 speculated on the existence of an esterase, active on cellobiose octaacetate [9]. A cellulose acetate-deacetylating microorganism for degradation of cellulosic was described by Yamauchi and Sakai in 1994 [41]. Sakai et al. [42] observed enzyme activity that released acetic acid from CA, when a culture supernatant from Neisseria sicca was incubated with this substrate. The synergistic action of acetylesterase and endoglucanase was demonstrated by incubating a CA of DS 0.7 exclusively with an endoglucanase and in parallel with an endoglucanase and an esterase (Fig. 5). Acting alone the endoglucanase required at least $72 \mathrm{~h}$ to fragment CA with DS 0.7 to a certain end. When both enzymes were present a drastic reduction in chain length occurred within the first hour, and no further reduction in chain length was seen after $24 \mathrm{~h}$. The synergy in CA degradation between endoglucanase and acetylesterase was also reported by Moriyoshi et al. [43, 44].

Ishigaki and coworkers [45] reported on a bacterial lipase from Bacillus sp. S2055, which was partly purified together with cellulase activity. Both enzymes together were able degrade CA plastic film of DS 1.7. The bacterial enzymes were compared in their ability to degrade CA with commercial lipases and esterases. It is interesting to note that none of the commercial enzymes were able to degrade CA. The lipase activity was tested with olive oil and the esterase activity with p-nitrophenyl acetate. Unfortunately none of the naturally occurring acetylated polysaccharides were used as reference substrates.

Altaner et al. (2003) [46] isolated an acetyl esterase from a commercial enzyme preparation. The enzyme released acetic acid from water-soluble and water-insoluble cellulose acetates, native and chemically acetylated xylan as well as acetylated starch. The acetyl esterase specifically 

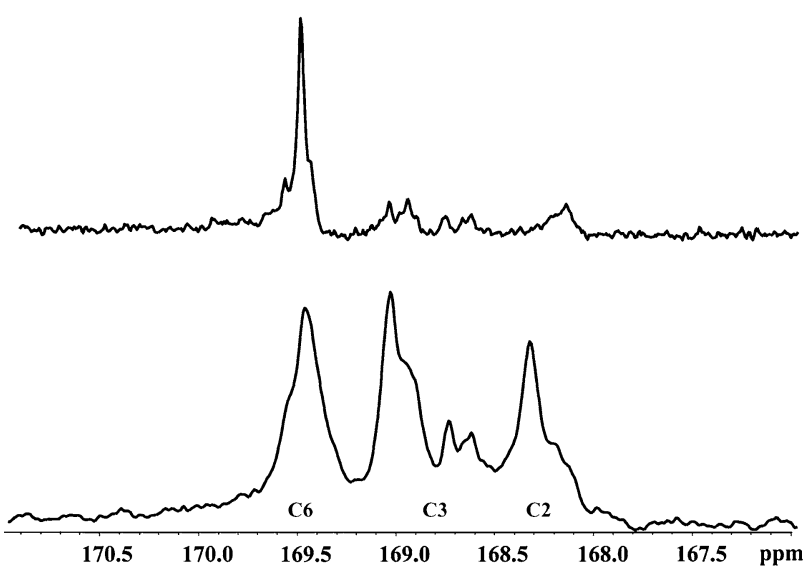

Fig. $6{ }^{13} \mathrm{C}$ NMR carbonyl signals of CA DS 0.7 (bottom) and after incubation with carbohydrate esterase family 1 enzyme from Aspergillus oryzae (top). Signals of acetyl substituents at C3 and C2 position are missing due to enzyme-aided deacetylation

cleaved off the acetyl substituents from the C2- and C3positions from CAs of DS $<1.8$ and left the acetyl substituents at the C6-positions intact without degrading the polysaccharide (Fig. 6). The acetyl esterase derived no advantage from the presence of an endoglucanase; it was able to function independently from endoglucanase activity.

Moriyoshi, et al. in 2005 coined the term cellulose acetate esterase, based on their study on an isolated esterase from Neisseria sicca [44]. The authors investigated the mode of action by incubation with the model compounds acetyl-B-D-glucopyranosides and -galactopyranosides, substituted in different positions. The main target of the enzyme was the position C-3 of the glucopyranoside, followed by position C-2. The positions C- 4 and C- 6 were deacetylated at slower rates. It is noteworthy that the deacetylation also included the C-6 position, which somehow justifies the term cellulose acetate esterase. When the 2,3,4,6-tetra- $O$-acetyl- $\beta$-D-galactopyranoside was incubated, the positions C-3 and C- 6 were preferably liberated from their acetyl substituents, and the enzyme exhibited clear regioselectivity in its deacetylation. The investigation was not extended by including polymeric CA.

Cellulose acetate was found to be a substrate of several acetyl xylan esterases (AXE) [47]. Eight AXEs from different carbohydrate esterase (CE) families were tested on their activity against CA with a degree of substitution of 0.7 and 1.4. The classification of the AXEs into CE families according to their structure by hydrophobic cluster analysis followed clearly their activity against CA. Within the same CE family similar and between the CE families different deacetylation behaviors could be observed. Furthermore, each esterase family showed a distinct regioselective mode of action. The CE1 family enzymes regioselectively cleaved the substituents in $\mathrm{C} 2-$ and $\mathrm{C} 3-$ position, while CE 5 family enzymes only cleaved the acetyl groups in C2-position. CE 4 family enzymes seemed to interact only with the substituents in C3-position. Evidence was found that the deacetylation reaction of the CE 1 family enzymes proceeded faster in C2- than in C3-position of CA. The enzymes were able to cleave acetyl groups from fully substituted anhydroglucose units.

In another study, the substrate specificity of selected enzymes classified under CE family 4 (CE4) have been examined. Chitin deacetylase from Mucor rouxii and both a native and a truncated form of acetyl xylan esterase from Streptomyces lividans were found to be active on both xylan and several soluble chitinous substrates (Fig. 7). An acetyl xylan esterase from Bacillus pumilus, classified under CE family 7, was found to be inactive towards all chitinous substrates tested [47].

In a combined view on the work of Altaner et al. [46] and Caufrier et al. [48], one could come to the conclusion that CE4 enzymes seem to be the most versatile esterases, since CE4 enzymes act as acetyl xylan esterases, as chitin deacetylases, and as cellulose acetate esterases, although they only liberate acetyl substitutents from C3. A closer look to the conformation of the artificially and naturally occurring acetylated polysaccharides, cellulose acetate, acetylxylan and chitin reveals that the acetyl substituents of these polysaccharides are identically arranged in the equatorial plane. Accordingly it would be conceivable that the active site of the same enzyme, specialized for deacetylation of naturally occurring acetylxylan and chitin, would also be able to accommodate cellulose acetate, in situations where it has been partly deacetylated before. This prior step could be achieved under the conditions of heat and high $\mathrm{pH}$, as they occur during composting or by photo degradation. Once a certain loss of acetyl substituents has occurred, naturally occurring microorganisms with extracellular enzyme systems designed by nature for cellulose, xylan, and chitin degradation can also utilize cellulose acetate.

Besides hydrolases like cellulases and acetylesterases, many microorganisms produce cellobiose oxidizing enzymes like quinone oxidoreductase (CBQ) and cellobiose oxidase $[49,50]$. During oxidation of cellobiose both enzymes reduce quinones to the corresponding phenols. Cellobiose oxidase also reduces $\mathrm{Fe}(\mathrm{III})$, together with hydrogen peroxide which is also produced by this enzyme, thus forming a Fenton's reagent resulting in a hydroxyl radical production [51].

\section{Photo Degradation}

Besides biological degradation, photo degradation is an important route for decomposing cellulose acetate 
Fig. 7 Identity of conformation and arrangement of substituents in cellulose acetate (top) and naturally acetylated polysaccharides like chitin (middle) and acetylxylan (XA, bottom)
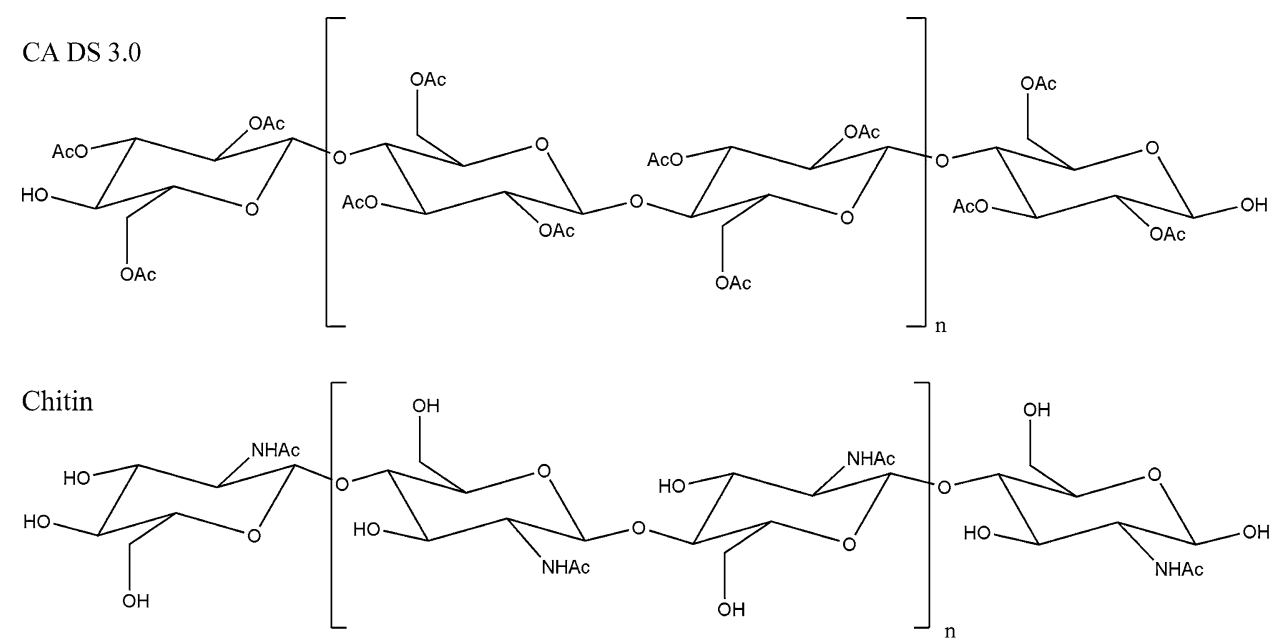

XA DS 2.0

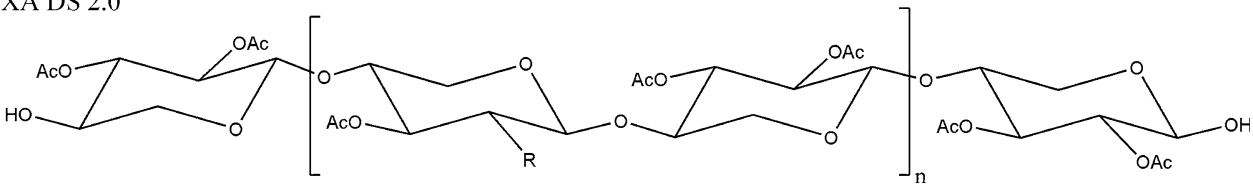

materials in the environment. There are different ways to induce photo degradation, which have in common the formation of radicals initiated by the absorption of light.

Cellulose acetate is made from raw materials with a high content of $\alpha$-cellulose, but still contains some impurities which might be responsible for the absorption of light in the far UV light region with wavelengths shorter than $280 \mathrm{~nm}$ range [52-54]. Cellulose acetate has an absorption at approximately $260 \mathrm{~nm}$ which is attributed to ketonic carbonyl groups [53]. In consideration of the fact, that the sunlight reaching earth's surface after being filtered by the atmosphere has a lower cut-off of approximately $300 \mathrm{~nm}$, one might conclude that cellulose acetate does not significantly photo degrade in a natural environment. This is only valid if other factors like air, water and contaminations are neglected. Secondary mechanisms are important in the photo degradation of cellulose acetate, which comprise other substances absorbing light and generating radicals for reacting with the cellulose acetate structure, these include photocatalytic oxidation or photosensitized degradation. The two latter routes can enhance the photo degradation of cellulose acetate significantly if suitable additives are applied. The degradation of pure cellulose acetate by irradiation with light in the far UV-range $(\lambda<300 \mathrm{~nm})$ and the regarding studies are helpful in obtaining an understanding of the general photo degradation mechanism.

\section{Degradation by Far UV-Light}

The degradation by direct irradiation of the solid material has been studied on powder, film and fibers. Several analytical methods have been applied to study degradation, for example, ESR spectroscopy for the identification of the radical intermediates, mass spectroscopy for the identification of decomposition products, viscosity measurement, and various measures of substitution degree.

Early studies of photo degradation investigated the parameters in deteriorating cellulosic materials and fibers for achieving a better photo stability. The photo degradation was analyzed under normal (oxygen containing) atmosphere with bright fibers (i.e. without pigments) by following the effects on the tensile strength [55], or with powders by following the effects on the intrinsic viscosity [52]. Under similar conditions the acetate fibers were more stable than rayon fibers. Note that Jortner [52] was not aware that deacetylation was a main result of the irradiation and this makes a bias in his calculation of the quantum yield from the results of viscosity measurements.

The studies by Hon [53] provided insight into the mechanisms, which comprises both a polymer chain scission of the glucosidic bond and a cleavage of side groups. Acetate fibers were irradiated in quartz tubes within the ESR cavity under vacuum at $77 \mathrm{~K}$ with far UV-light. The ESR spectra were recorded at different temperatures which allow one to differentiate the individual signals due to the different stabilities and decays of the radicals. By this method four different radicals were identified: the methyl radical as results of the cleavage of the acetate side groups and the three radical types which are generated also from the irradiation of un-substituted cellulose: the alkoxy radical at $\mathrm{C}_{4}$ and the radical at $\mathrm{C}_{1}$ from the cleavage of the glucosidic bonds and also the radicals from hydrogen abstraction from ring-carbons. 
The analyses of the gaseous products (methane, carbon monoxide and dioxide) and the analyses of the DS confirmed and supported the interpretation, that the cleavage of the side groups is playing a major role in the direct photodegradation. Interestingly, during the irradiation the degree of substitution decayed initially rapidly up to a level of $30 \%$ bound acetic acid which corresponds to a degree of substitution of approximately 1 and slows down thereafter, see Fig. 8 .

If the irradiation was performed through a glass window with near UV-light (>340 nm) only, no ESR signal could be detected and the fibers were stable and materials remained nearly unchanged (tensile strength, intrinsic viscosity, weight loss).

The interpretation of the mechanism with the initial free-radical intermediates was confirmed by Merlin and Fouassier in 1982 who performed similar ESR studies on acetate films [56] and compared the resulting spectra with those from cellulose and similar monomeric reference substances.

\section{Degradation by Near UV-Light: Use of Photo}

Sensitizers

Although far-UV light which is able to deteriorate pure cellulosic materials is not present in normal sunlight, the use of photosensitizers will allow and enhance the degradation with near UV-light. These photosensitizers are acting either by energy transfer or by initial radical abstraction.

Endo and coworkers from the Yamagata University explored the use of different additives, as photo sensitizers and so called photo acid generators on the photo degradation of cellulose acetates [54, 57-59]. Acetate films (40 $\mu \mathrm{m}$ thickness) containing different additives in various

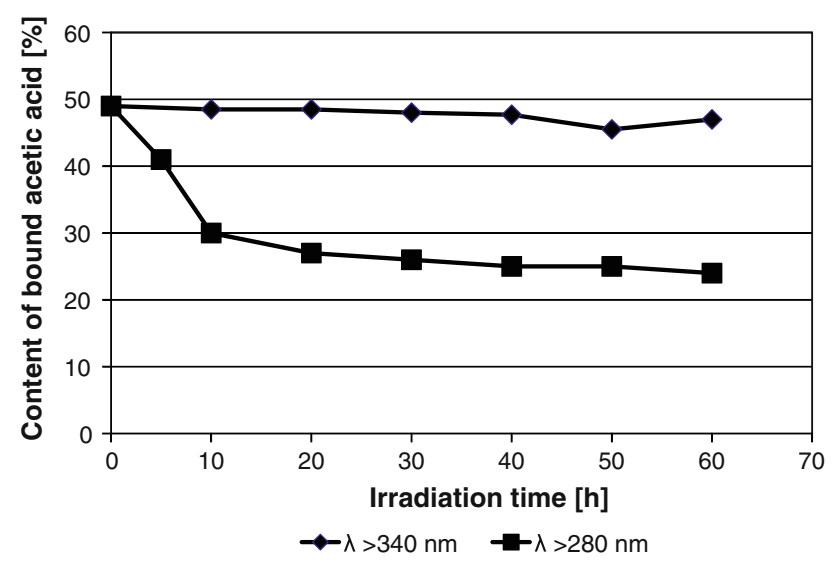

Fig. 8 Decrease of bound acetic acid content of cellulose acetate fibers irradiated with far $(\lambda>280 \mathrm{~nm})$ and near UV light $(\lambda>340 \mathrm{~nm})$, taken from Fig. 7 in [53] (recalculated from bound acetic acid with corrections) concentrations were irradiated in glass vials with a xenon arc light $(\lambda>275 \mathrm{~nm})$ simulating solar exposure.

The irradiations of the photosensitizer benzophenone in concentration of $1-16 \%$ embedded in acetate films were examined with various analytical techniques [54]. The yield of the decomposition products of $\mathrm{CO}, \mathrm{CO}_{2}$, and acetic acid increased with higher concentration of benzophenone in the film. The decrease of the polymeric molecular weight and the increase of the polydispersity indicated the scission of the glucosic bonds [59]. The authors concluded that the irradiation starts with the hydrogen abstraction from the polymer by the excited triplet state in benzophenone, and passes through similar radical intermediates as by far UV-light (Fig. 9).

Additional additives explored by this group were so called "photoacid generators," substances which generate strong acids after irradiation (triphenylsulphonium trifluoromethanesulfonate (TPS) [57], and diphenyliodonium trifluoromethynsulfonate (DPI) [58] which were embedded in CA films and irradiated with UV light $(\lambda>275 \mathrm{~nm})$ for the release of sulfonic acid. DPI was more effective than TPS and contributes to an effective release of acetic acid after irradiation [57, 59] and could further be enhanced by the use of benzophenone, which acts as triplet state sensitizer for DPI.

\section{Degradation by Near UV-Light: Catalytic Photo Oxidation}

The most common photo catalyst is titanium dioxide. Commercial $\mathrm{TiO}_{2}$ pigments are manufactured in two morphological crystalline forms (anatase or rutile). The non-modified anatase form exhibits photo oxidative reactivity at irradiation up to $390 \mathrm{~nm}(\sim 3.2 \mathrm{eV})$. Photocatalysts based on titanium dioxide are meanwhile broadly used for different applications, such as water purification, water cleavage, air cleaning or even "self" cleaning of surfaces [60].

Pigments of titanium dioxide with a particle size of $0.2-0.3 \mu \mathrm{m}$ are the common delustering and whitening agents in fibers and in plastic materials. For these applications, with some outdoor exposure, these pigments need to be modified to reduce their activity which is done by dense coating of the surface of the pigments with inert oxides, by inclusion of other elements in the crystalline matrix of titanium dioxide, or by doping of different elements on the pigment surface. Without these modifications the anatase pigment is responsible for oxidative degradation which is understood as radical chain reaction after electron transfer for which the semi-conductor model is usually used [61, 62].

The photo activity of $\mathrm{TiO}_{2}$ types has been extensively studied [55, 60, 62-65]. Figure 10 provides a description of 
Fig. 9 Scheme of photo sensitized degradation of cellulose acetate (CA) according to [54]
(1) $\mathrm{Ph}_{2}-\mathrm{C}=\mathrm{O} \longrightarrow \mathrm{hv} \longrightarrow\left[\mathrm{Ph}_{2}-\mathrm{C}=\mathrm{O}\right]^{\top}-+\mathrm{CA} \longrightarrow \mathrm{Ph}_{2}-\mathrm{C}^{\bullet}-\mathrm{OH}+\mathrm{CA}^{\bullet}$

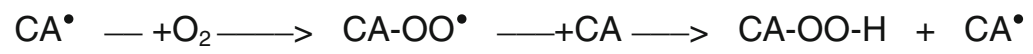
$\mathrm{CA}-\mathrm{OH}$
Fig. 10 Schematic description of the photo oxidation mechanism of anatase titanium dioxide in the semiconductor model according to [61]

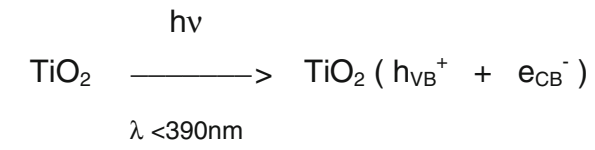

$\mathrm{e}^{-}+\mathrm{TiO}_{2}-\mathrm{O}_{2 \mathrm{ds}}$

$\mathrm{TiO}_{2}+\mathrm{O}_{2}-$

$\longrightarrow+\mathrm{H}^{+} \longrightarrow \mathrm{HO}_{2}$.
$\mathrm{h}_{\mathrm{VB}^{+}}^{+}+\mathrm{TiO}_{2}-\mathrm{H}_{2} \mathrm{O}_{\mathrm{ads}} \longrightarrow \mathrm{TiO}_{2}-\mathrm{H}^{+}+\mathrm{HO}$.

$\mathrm{CA}+\mathrm{HO} \cdot / \mathrm{HO}_{2} \cdot$
$-+\mathrm{O}_{2} \longrightarrow \longrightarrow$ CA-O-O-H .... the photo oxidation mechanism of anatase titanium dioxide in the semiconductor model according to [61]. Irradiation of the anatase crystal with near UV-light $(<390 \mathrm{~nm})$ excites electrons from the valence band in the conductivity band (1); which could reduce oxygen by electron transfer (2) and the remaining "holes" in the valence band $\left(\mathrm{h}_{\mathrm{VB}}^{+}\right)$ might oxidize water adsorbed on the surface (3) generating various radicals. These formed radicals might start any degradation of the surrounding polymer by hydrogen abstraction with subsequent radical chain reaction of the polymer, particularly at higher temperatures if the activation energy for the cleavage of peroxides is achieved.

The influence of temperature and humidity on the titanium dioxide catalyzed degradation of acetate yarns was studied by Egerton et al. [55] who found that higher temperatures and particularly a high humidity will enhance the degradation. These findings can be understood with the above mentioned mechanism which includes the temperature required for the cleavage of peroxides in the chain radical mechanism, the moisture adsorbed on the titanium dioxide surface to be oxidized, and to avoid the recombination of the excited states.

Degradation catalyzed by titanium dioxide pigments can be identified with microscopy as shown by the holes in films, see Fig. 11a, or fibers irradiated in a weatherometer (see Fig. 11b).

Haynes and coworkers [66] studied the photo degradation of cigarette filters with a conventional $\mathrm{TiO}_{2}$ pigment. The filters were placed in wired mesh cages a few inches above a flat roof in Tennessee, where they were exposed to sun, wind and rain. There was no soil contact or standing water in this study. Although the biodegradation was minimized, a weight loss between 10 and $20 \%$ was recorded after the first year, and 20 to $40 \%$ weight loss was measured after 2 years. The DS and molecular weight determinations of exposed CA fiber samples showed that the polymer was partly deacetylated, and its molecular weight was decreased during the photo degradation process.

A working group of CORESTA (Centre de Coopération pour les Recherches Scientifiques relatives au Tabac) developed an accelerated test to monitor the degradability of cigarette filters, which was described by Brodof [67]. A commercial bench-top near-UV weathering instruments was used in which the butts could be soaked with water in defined intervals and the degradation was followed by weight determinations.

Various ideas have been disclosed to utilize and enhance the photoactivity of $\mathrm{TiO}_{2} \mathrm{~s}$. In 1993, Irick showed that surface treatment of pigmentary anatase $\mathrm{TiO}_{2}$ with barium phosphate could provide enhanced photodegradation [68].

The photo reactivity of titanium dioxide pigments can also be improved by using nano-structured ultrafine anatase pigments which have a much larger specific surface area. This approach has been used in acetate films [69] and fibers [70]. Jang demonstrated that ultrafine titanium dioxide could be used in acetate films for enhancing the enzymatic degradation utilized in biodegradation [69]. Brodof used 


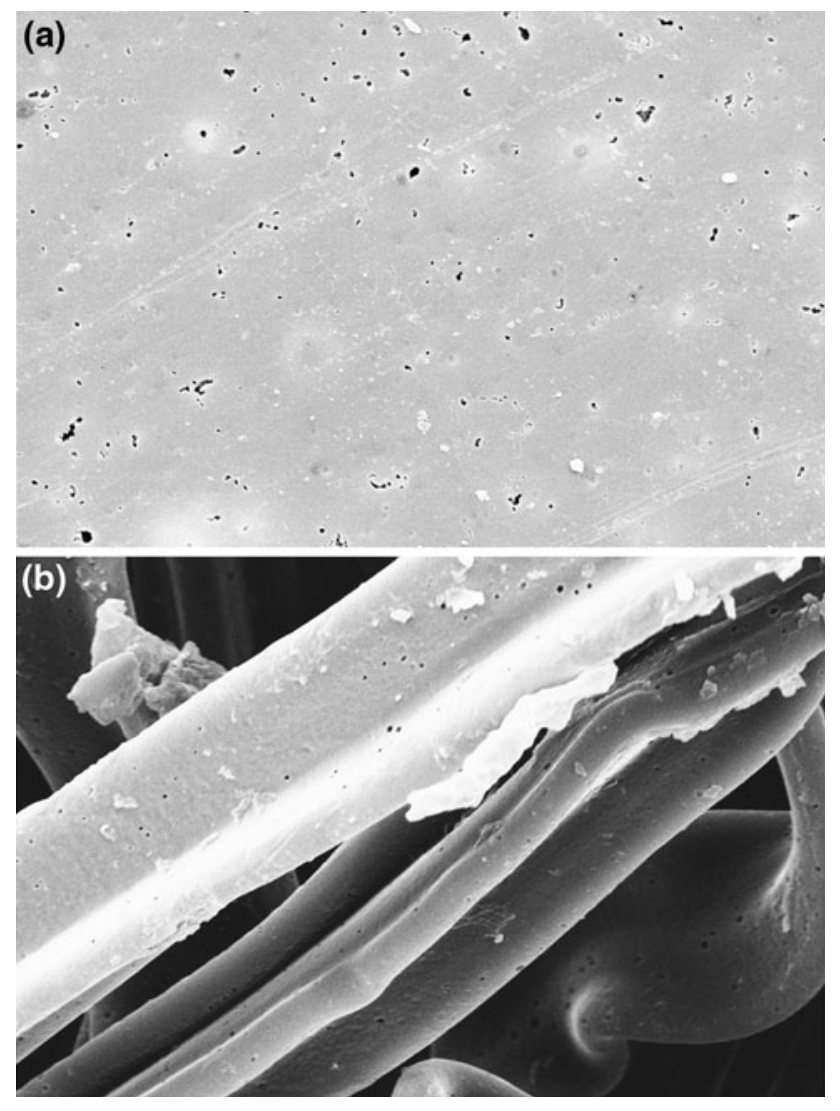

Fig. 11 a Photo micrograph of CA film with $\mathrm{TiO}_{2}$ pigments irradiated in a weatherometer with near UV light. b Scanning Electron Microscopy (SEM) of acetate fibers from cigarette filters after few months outdoor exposure

ultrafine titanium dioxide in fibers and demonstrated the enhanced degradation in the accelerated test and under outdoor exposure conditions. In the humid and sunny climate of South Florida a weight loss of $65 \%$ for acetate cigarette filters (without wrapping paper) could be achieved within 6 months exposure using $0.7 \%$ ultrafine titanium dioxide in the fibers, compared to $17 \%$ for the standard material [70, 71].

Itoh et al. have also evaluated the use of $\mathrm{TiO}_{2}$ to enhance CA degradation with a $\mathrm{DS}<2.15$ adding $\mathrm{TiO}_{2}$ with particle sizes 0.01 to $1 \mu \mathrm{m}$ which will provide more degradable material [72]. In US Patent 5,804,296, Itoh et al. disclose the benefits of CA fibers with high surface area $\mathrm{TiO}_{2}$ additives, $\left(\geq 50 \mathrm{~m}^{2} / \mathrm{g}\right)$ that provide enhanced photo degradation rates [73].

\section{Mechanism Synergy and Disintegration}

In the outdoors environment, the degradation of CA materials can include multiple mechanisms. Deacetylation seems to be the first step in both, photochemical and biological degradation [69]. In cases where the DS is too high for esterases, the first step of biodegradation can be initiated by UV light irradiation or by chemical hydrolysis, with the latter being subject to $\mathrm{pH}$ change. Also in already partly hydrolyzed CA, like commercial acetate with a DS of 2.5 , the critical deacetylation step can be accelerated by non-biological mechanisms under the influence of sunlight, temperature and water. With decreasing DS there is a better chance for biological systems to attack CA, especially if the DS reaches a region of about 1.5 to 1.8 . Acetyl esterases, designed by nature for acetylxylan and chitin biodegradation, are the first enzymes which remove the acetyl substituents until a DS $\sim 1$ is reached. When the DS approaches a region $\leq 0.9$, CA becomes water-soluble and the polymer readily disperses [2]. In addition, at this point CA becomes available to endoglucanases, shortening the CA chain in a random fashion preferably at those regions where acetyl substituents do not hinder the contact to the substrate. A synergistic process between acetylesterases and endoglucanases starts with the result of a quick liberation of free sugars and acetic acid.

Since deacetylation is the rate determining step in biodegradation of CA, followed by the comparatively faster enzymatic hydrolysis of the cellulose backbone, the overall DS of the remaining material will stay nearly unchanged during degradation. But the specific surface area of the material increases with ongoing degradation, due to pitting caused by different rates of degradation at different spots depending on local conditions at micro scale. This increased surface area exposed to environment and accessible for microorganisms facilitates further degradation [69].

One approach to enhancing biodegradation is to add a water soluble or dispersible additive. Ito et al., in US patent 5,913,311 describe ideas for this approach for making CA fibers containing various water soluble materials including hydroxypropyl cellulose, polyethylene glycol, starch, and dextrin with the expectation that when the fibers are placed in water the additive(s) would dissolve away and increase the fibers surface area [74].

$\mathrm{CA}$ is known to naturally hydrolyze at rates that are dependent on the temperature and moisture level [75]. Decomposition accelerating agents added to CA fibers can serve to facilitate the chemical hydrolysis of CA. These concepts are challenging to implement, because one wouldn't want the CA material to degrade before the polymer has been discarded and the material should have the same qualities compared to the non-modified polymer. To be successful, the overall formulation may have decomposition accelerating agents combined with a reaction controlling agent, which stabilizes the material before disposal. Yamashita evaluated the addition of using various 
acids to CA films for enhancing the biodegradation [76]. In US Patent 6,571,802 Yamashita proposed combining the acid with a stabilizing agent which releases the acid upon contact with water, thus preventing the additives from deacetylating the $\mathrm{CA}$ and generating acetic acid before disposal [77]. Robertson in US Patent Application 2009/ 0151738 disclosed the idea of making an enhanced degradable cigarette filter by adding a hydrolysis causing additive to the filter. The idea is said to require the use of a coating or pill to hold the hydrolysis agent until the filter is discarded, then the additive would be released upon contact with water [78].

In US Patent 6,133,439 Buchanan et al. described environmentally non-persistent cellulose ester fibers based upon using a low DS polymer ( $\mathrm{DS}=2.0-2.2$ ) and anatase $\mathrm{TiO}_{2}$ [79]. The benefit of this blend was to enhance both biodegradation and photodegradation in fibers simultaneously and allow synergy between the two mechanisms. Ishigaki et al. explored this synergy further by evaluating the benefits of UV irradiation on the enzymatic degradation of CA fibers without the presence of a photocatalyst like $\mathrm{TiO}_{2}$ [80]. The UV light resulted in a decrease in the molecular weight of CA, but did not affect the DS. The removal of the lower molecular weight material allowed an increase in surface area, and thus better assess for the cellulase enzyme. Jang et al. [69] studied the effect of cellulase degradation on films with anatase $\mathrm{TiO}_{2}$ nanoparticles with UV irradiation. They found that the UV light significantly changed the surface properties by increasing the hydrophilicity and lowering the DS. Both these changes are believed to enhance the enzymatic degradation.

In addition to mixed modes of degradation, one could choose optimum product designs to enhance disintegration. A large amount of CA polymer is used to make cigarette filters, which are often discarded and become litter. Disintegration can be influenced by many factors including: rainfall, sunlight, biological agents, temperature, atmospheric oxidants, air pollutants, and mechanical agitation. Various ideas for enhancing smoke filter disintegration have been patented. Rapid disintegration is a key step in the disappearance of a discarded litter item, and it also allows enhanced degradation rates. For example, rapid water disintegration can be designed directly into consumer products, and thus reduce visible litter while enhancing degradation [81-86].

The disintegration of conventional filter cigarettes is impeded due to the highly entangled network of the fibers, plus the structure is reinforced by the addition of plasticizers fusing the fibers together. When investigating the effect of triacetin plasticizer on degradation Haynes and coworkers came to the conclusion that there was no effect on the percentage weight loss of the filter due to the presence or absence of the plasticizer when exposed to the environment [66]. There was only a visible difference in appearance between the two samples. The sample without the plasticizer had a less defined shape resembling a discarded cotton ball.

Early disclosures of the concept of a disintegratable cigarette filter were in US Patents 4,022,740 and 4,074,724 by Morie and Sloan. The concept was to bond the individual fibers in a filter with water soluble polyester polymers which would dissolve upon prolonged exposure to water and release individual fibers [81, 82]. Tsugaya et al. [83] disclosed in US Patent 5,947,127 the bonding of individual fibers with particulate hot-melt adhesive in place of conventional plasticizers, thus allowing water disintegratability.

In 1996, new filter designs were disclosed that would allow enhanced filter disintegration when exposed to water [84]. In the absence of plasticizer, a bundle of CA fibers could be made less entangled by reducing the fiber lengths, fiber crimp level, and increasing the individual fiber size. One approach is to reduce the entanglement by constructing a multi-segmented filter consisting of short cut segments instead of a single filter segment. This minimum size of a short cut filter segment is roughly $5 \mathrm{~mm}$ for a $7 \mathrm{~mm}$ diameter filter, and research has shown that complete water dispersibility is possible with 8-denier per filament fibers at $5-\mathrm{mm}$ lengths. When the denier of the individual fibers is increased, fibers become stiffer, reducing the amount of fiber entanglement. In addition to fiber length, it has been shown that reducing the crimp level is another approach to improve the mechanical disintegration of discarded cigarette filters. Another approach disclosed by Wilson and Harris [85] was to perform a series of partial cuts on a filter which would result in the individual fibers all being short cut, while the filter is not cut completely. This approach provides the advantage that shorter fiber lengths can be cut, thus allowing smaller denier-per-filament fibers which would reduce cost and allow optimal filter performance. The filter making process for producing partial cut filter is not commercial, but has been envisioned and disclosed [86].

A filter making process which constructs filter from short-cut randomly-oriented fibers was disclosed by Berger and Sproull in 1972 [87]. Although filter disintegration was not the intention of the design, Clarke and Araujo disclosed that filter made using the randomly-oriented fiber process would be twice as likely to degrade $(46 \%)$ after 1 year of weathering versus conventional filters degradation (23-30\%) [88].

This review highlights that CA polymer based consumer products have good potential for environmental degradation. There is also potential for increasing the degradation with accelerated disintegration by redesigning commercial products. 
Acknowledgments This research was supported by the Global Acetate Manufacturers Association (GAMA) as a group project with support from all acetate manufacturers.

Open Access This article is distributed under the terms of the Creative Commons Attribution Noncommercial License which permits any noncommercial use, distribution, and reproduction in any medium, provided the original author(s) and source are credited.

\section{References}

1. Edgar K (2004) Organic cellulose esters. In: Mark HF (ed) Encyclopedia of polymer science and technology, vol 9, 3rd edn. Wiley, New York, pp 129-158

2. Rustemeyer P (2004) Macromol Symp 208:1-394

3. Potts JE, Clendinning RA, Ackart WB (1972) An investigation of the biodegradability of packaging plastics. EPA Study EPA-R272-046

4. Buchanan CM, Gardner RM, Komarek RJ (1993) J Appl Polym Sci 47:1709-1719

5. Komarek RJ, Gardner RM, Buchanan CM, Gedon S (1993) J Appl Polym Sci 50:1739-1746

6. Gardner RM, Buchanan CM, Komarek R, Dorschel D, Boggs C, White AW (1994) J Appl Polym Sci 52:1477-1488

7. Rivard CJ, Adney WS, Himmel ME, Mitchell DJ, Vinzant TB, Grohmann K, Moens L, Chum H (1992) Appl Biochem Biotechnol 34-35:725-736

8. Gu J-D, Eberiel DT, McCarthy SP, Gross RA (1993) J Environ Polym Degr 1:143-153

9. Reese ET (1957) Ind Eng Chem 49:89-93

10. Nuttall FE, Cantor PA (1968) US Patent 3,398,834

11. Cantor PA, Mechalas BJ (1969) J Polym Sci Part C 28:225-241

12. Ho LCW, Martin DD, Lindemann WC (1983) Appl Environ Microbiol 45:418-427

13. Northrop DM, Rowe WF (1987) Effect of the soil environment on the biodeterioration of man-made textiles. In: Llewellyn GC, O'Rear CE (eds) Biodeterioration research 1. Plenum Press, New York, pp 7-16

14. Gu J-D, Eberiel D, McCarthy SP, Gross RA (1993) J Environ Polym Degrad 1:281-291

15. Nelson M, McCarthy SP, Gross RA (1992) Polym Mater Sci Eng 67:139-140

16. Gu J-D, Yang S, Welton R, Eberiel DT, McCarthy SP, Gross RA (1994) J Environ Polym Degrad 2:129-135

17. Sakai K, Yamauchi T, Nakasu F, Ohe T (1996) Biosci Biotechnol Biochem 60:1617-1622

18. Gartiser S, Wallrabenstein M, Stiene G (1998) J Environ Polym Degrad 6:159-173

19. Zenjian L, Hibi T, Hamano Fl, Shibata T, Furuichi K (2003) Preprints of the 10th annual meeting of the Cellulose Society of Japan, p 97

20. Edgar KJ, Buchanan CM, Debenham JS, Rundquist PA, Seiler BD, Shelton MC, Tindall D (2001) Progr Polym Sci 26:1605-1688

21. Körner I, Stegmann R (1998) Acta Hortic 469:97-110

22. Rhodia customer support information 3/97-(1997) The biological degradability and compostability of Rhodia filter tow. http://www.acetateweb.com/techinfo.htm. Accessed on 18 June 2010

23. van der Zee M, Stoutjesdijk JH, Feil H, Feijen J (1998) Chemosphere 36:461-473

24. Ach A (1993) JMS-Pure Appl Chem A 30:733-740

25. Yoshioka M, Shiraishi N (2000) Mol Cryst Liq Cryst 353:59-73
26. Teufel E, Wilmund R (1995) US Patent $5,427,852$

27. Willmund R (1995) US Patent 5,462,801

28. Teufel E, Wilmund R (1996) US Patent 5,495,860

29. Cannon JN (1996) US Patent 5,512,230

30. Buchanan CM, Dorschel D, Gardner RM, Romarek RJ, Matosky AJ, White AW, Wood MD (1996) J Environ Polym Degrad 4:179-195

31. Itoh M, Kiyose A, Hirao K, (1995) US Patent 5,478,386

32. Divne C, Stahlberg J, Reinikainen T, Ruohonen L, Pettersson G, Knowles JKC, Teeri TT, Jones TA (1994) Science 265:524528

33. Knowles JKC, Lentovaara P, Murray M, Sinnott ML (1988) J Chem Soc Chem Commun 21:1401-1402

34. Rouvinen J, Bergfors T, Teeri T, Knowles JKC, Jones TA (1990) Science 249:380-386

35. Itävaara M, Siika-aho M, Viikari L (1999) J Environm Polym Degrad 7:67-73

36. Montenecourt BS, Eveleigh DE (1983) Trends Biotechnol $1: 156-161$

37. Spezio M, Wilson DB, Karplus PA (1993) Biochemistry 32:9906-9916

38. Puls J, Horner S, Kruse Th, Saake B, Heinze T (1998) Das Papier 52:743-748

39. Karlsson J, Momcilovic D, Wittgren B, Schülein M, Tjerneld F, Brinkmalm G (2002) Biopolymers 63:32-40

40. Saake B, Horner St, Puls J (1998) ACS Symp Ser 688:201216

41. Yamauchi T, Sakai K (1994) JP 07289243 Teijin Ltd, Japan, Osaka

42. Sakai K, Yamauchi T, Nakasu F, Ohe T (1996) Biosci Biotechnol Biochem 60:1617-1622

43. Moriyoshi K, Ohmoto T, Ohe T, Sakai K (2003) Biosci Biotechnol Biochem 67:250-257

44. Moriyoshi K, Yamanaka H, Ohe T, Sakai K (2005) Biosci Biotechnol Biochem 69:1292-1299

45. Ishigaki T, Sugano W, Ike M, Fujita M (2000) J Biosci Bioeng 90:400-405

46. Altaner C, Saake B, Puls J (2003) Cellulose 10:85-95

47. Altaner C, Saake B, Tenkanen M, Eyzaguirre J, Faulds CB, Biely P, Viikari L, Siika-aho M, Puls J (2003) J Biotechnol 105:95-104

48. Caufrier F, Martinou A, Dupont C, Bouriotis V (2003) Carbohydr Res 338:687-692

49. Eriksson K-E, Pettersson B, Westermark U (1974) FEBS Lett 49:282-285

50. Ayers AR, Ayers SB, Eriksson K-E (1978) Eur J Biochem 90:171-181

51. Ander P (1994) FEMS Microbiol Rev 13:297-312

52. Jortner J (1959) J Polym Sci 37:199-214

53. Hon NS (1977) J Polym Sci 15:725-744

54. Hosono K, Kanazawa A, Mori H, Endo T (2007) J Appl Polym Sci 105(6):3235-3239

55. Egerton GS, Shah KM (1968) Tex Res J 38(2):130-135

56. Merlin A, Fouassier JP (1982) Angew Makromol Chem 108:185-195

57. Hosono K, Kanazawa A, Mori H, Endo T (2006) J Adhes Soc Jpn 42:350-355

58. Hosono K, Kanazawa A, Mori H, Endo T (2007) Cellulose 14:529-537

59. Hosono K, Kanazawa A, Mori H, Endo T (2008) J Appl Polym Sci 109:3157-3164

60. Carp O, Huisman CL, Reller A (2004) Prog Solid State Chem 32:33-177

61. Allen NS (1994) Photofading and light stability of dyed and pigmented polymers. Polym Degrad Stab 44:357-374

62. Rabek JF (1995) Polymer photodegradation-mechanisms and experimental methods. In: Chapter 5-Role of metal compounds 
in the photodegradation of polymers, Chapman and Hall, New York, pp. 353-365

63. Irick G Jr (1972) J Appl Polym Sci 16:2387-2395

64. Linsebigler AL, Guangquan 1, Yates JT Jr (1995) Chem Rev 95:735-758

65. Allen NS, McKellar JF (1980) Photosensitized degradation of polymers by dyes and pigments. In: Photochemistry of dyed and pigmented polymers. Elsevier Science and Technology, p 247

66. Haynes SK, Wilson SA, Strickler DV (1991) Study of the environmental degradation of cigarette filters: a simulation of the roadside or parking lot environment. Eastman Chemical customer service technical report. http://www.acetateweb.com/techinfo. $\mathrm{htm}$. Accessed on 18 June 2010

67. Brodof TA (1999) Tobacco reporter: $82-85$

68. Irick G Jr (1993) US 5,242,880

69. Jang J, Lee HS, Lyoo WS (2007) Fibers Polym 8:19-24

70. Brodof TA, Hopkins JB Jr (1996) US Patent 5,491,024

71. Brodof TA, Hopkins JB Jr (1997) US Patent 5,647,383

72. Itoh M, Kiyose A, Hirao T (1998) US Patent 5,720,803

73. Itoh M, Miyazawa A, Aoe T, Ikemoto 0, (1998) US Patent $5,804,296$

74. Ito K, Matsumoto T, Tokida A, Shibata T, Yamashita Y, (1999) US Patent 5,913,311
75. Vos KD, Burris FO, Riley RL (1966) J Appl Polym Sci 10: $825-832$

76. Yamashita Y, Endo T (2005) J Appl Polym Sci 98:466-473

77. Yamashita Y (2003) US Patent $6,571,802$

78. Robertson RM, Thomas WC (2009) US Patent Appl 2009/ 0151738

79. Buchanan CM, Gardner RM, Harris JE, Irick G, Strickler DV (2000) US Patent 6,133,439

80. Ishigaki T, Sugano W, Ike M, Taniguchi H, Goto T, Fujita M (2002) Polym Degrad Stab 78:505-510

81. Morie GP, Sloan CH (1977) US Patent 4,022,740

82. Morie GP, Sloan CH (1978) US Patent 4,074,724

83. Tsugaya H, Taniguchi H, Oji N, Nishimura K (1999) US Patent $5,947,127$

84. Research Disclosure (Eastman Chemical) (1996) Environmentally Disintegradable Cigarette Filters. 386: 375-377

85. Wilson SA, Harris JE (1999) US Patent 5,947,126

86. Garthaffner MT (2008) US Patent 7,435,208

87. Berger RM, Sproull RC (1972) US Patent 3,658,626

88. Clarke PF, Araujo DS (2009) Patent Appl WO 2009/093051

89. MacKenzie LF, Sulzenbacher G, Divne C, Jones TA, Wöldike HF, Schülein M, Withers SG, Davies GJ (1998) Biochem J 335:409-416 